\title{
EFECTO DE DIFERENTES INOCULANTES SOBRE LA ACTIVIDAD MICROBIANA EN LA RIZÓSFERA DEL CULTIVO DE PALLAR (Phaseolus lunatus var. sieva) EN CONDICIONES DE CAMPO
}

\section{EFFECT OF DIFFERENT BIOINOCULANTS ON THE MICROBIAL ACTIVITY IN THE RHIZOSPHERE SOIL OF Phaseolus lunatus var. sieva UNDER FIELD CONDITIONS}

\author{
Elena Ramos Vásquez ${ }^{1}$ y Doris Zúñiga Dávila ${ }^{1}$
}

\begin{abstract}
Resumen
Se evaluó la actividad microbiana en la rizósfera de Phaseolus lunatus var. sieva, a partir de muestras de suelo colectadas en campo durante los diferentes estados fenológicos del cultivo, cuyas semillas fueron inoculadas con Bradyrhizobium sp. cepa PCYGIVN3, cepa PSNC4N2 y la interacción de éstas; además, se evaluó la aplicación de fertilizante nitrogenado N-P-K y el control sin inocular. Se determinaron la respiración y la actividad deshidrogenasa de los microorganismos en la rizósfera, las poblaciones microbianas y la materia seca vegetal de las plantas ensayadas.

La mayor producción de $\mathrm{CO}_{2}$ se observó durante la floración. La respiración en la rizósfera inoculada con la interacción de cepas fue mayor que la cepa PSNC4N2 (0.121 y $0.088 \mathrm{mg} \mathrm{CO} \cdot \mathrm{C}^{-}$ ${ }^{1} \cdot \mathrm{h}^{-1}$ respectivamente); siendo la primera significativamente mayor respecto al testigo y al tratamiento químico nitrogenado $\left[0.085 \mathrm{mg} \mathrm{CO}_{2} \cdot \mathrm{g}^{-1} \cdot \mathrm{h}^{-1}\right](\mathrm{p}<0.1)$. La actividad deshidrogenasa de la rizósfera inoculada con Bradyrhizobium cepa PSNC4N2 > cepa PCYGIVN3 > interacción, con 61.04, 56.57 y $51.37 \mu \mathrm{g}$ formazán $\cdot \mathrm{g}^{-1} \cdot(24 \mathrm{~h})^{-1}$ respectivamente, evidenciando diferencias significativas con la fertilización química nitrogenada [40.87 $\mu \mathrm{g}$ formazán $\left.\cdot \mathrm{g}^{-1} \cdot(24 \mathrm{~h})^{-1}\right]$. Los valores más altos de actividad deshidrogenasa se registraron antes de la cosecha, probablemente por la influencia ambiental y la presencia de fitonemátodes en las raíces. La actividad microbiana se presentó como una técnica más sensible que el recuento de microorganismos y permitió una buena correlación con los resultados de materia seca obtenidos en el tratamiento de la interacción de cepas de Bradyrhizobium y el inoculado con la cepa PSNC4N2, que presentaron los valores más altos de materia seca durante el periodo comprendido entre la floración y madurez de vainas.

Palabras clave: Actividad microbiana, Phaseolus lunatus var. sieva, producción de $\mathrm{CO}_{2}$, actividad deshidrogenasa
\end{abstract}

\begin{abstract}
Microbial activity was evaluated in Phaseolus lunatus var. sieva rhizosphere soil, during different vegetative growth stages of the crop under field conditions. Seeds were inoculated with Bradyrhizobium sp. strains PCYGIVN3, PSNC4N2 and the interaction of both. Nitrogen fertilizer (N-P-K) and a control without inoculation were also evaluated. Respiration and dehydrogenase activity of microorganisms inhabiting the rhizosphere soil, microbial populations and plant dry weight were determined.

The highest $\mathrm{CO}_{2}$ production rate was determined during flowering. Respiration from the rhizosphere soil inoculated with the interaction of both strains was higher than strain PSNC4N2 (0.121 and $0.088 \mathrm{mg} \mathrm{CO} \cdot \mathrm{g}^{-1} \cdot \mathrm{h}^{-1}$ respectively); the interaction was also significatively higher than the control and the nitrogenous chemical treatment $\left[0.085 \mathrm{mg} \mathrm{CO} \cdot \mathrm{g}^{-1} \cdot \mathrm{h}^{-1}\right](\mathrm{p}<0.1)$. Dehydrogenase activity from rhizosphere soil inoculated with Bradyrhizobium strain PSNC4N2 > strain PCYGIVN3 > interaction of both, with 61.04, 56.57 and $51.37 \mu \mathrm{g}$ formazan $\cdot \mathrm{g}^{-1} \cdot(24 \mathrm{~h})^{-1}$ respectively, and showed differences with respect to the chemical nitrogen fertilization [40.87 $\mu \mathrm{g}$ formazan $\left.\cdot \mathrm{g}^{-1} \cdot(24 \mathrm{~h})^{-1}\right]$. Higher values of dehydrogenase activity were reported before the harvest, probably due to the influence of the environment and the phytonematode infection of roots. Microbial activity was a more sensitive method than microorganism counting, showing a good correlation with plant dry weight obtained by the interaction of strains treatment and that inoculated with strain PSNC4N2. Both showed the highest dry matter values between flowering and pods maturity stages.
\end{abstract}

Key words: Microbial activity, Phaseolus lunatus var. sieva, $\mathrm{CO}_{2}$ production, dehydrogenase activity 
Enero - Diciembre 2008

\section{Introducción}

El uso de fertilizantes y pesticidas es una práctica agrícola muy frecuente que tiene como desventaja el alto costo que significa para el agricultor, además de traer como consecuencia la alteración del medio ambiente. Su reducción progresiva solo será posible mediante el empleo de tecnologías limpias, como el uso de inoculantes, aplicados sobre el sustrato edáfico sobre el cual se desarrollan las plantas, o sobre las semillas antes de la siembra. Su metodología constituye uno de los sistemas biotecnológicos con mayor futuro para la mejora de la producción vegetal, evitando los efectos secundarios de los métodos actuales de fertilización y promoviendo la protección frente a plagas. Ejemplo de este método es el establecimiento de simbiosis entre bacterias de los géneros Rhizobium y Bradyrhizobium con leguminosas (Defreitas et al., 1993; Andreeva et al., 1993; Probanza et al., 1997).

La prometedora perspectiva del uso de Rhizobium, Bradyrhizobium y PGPRs (Plant Growth Promoting Rhizobacteria) en cualquiera de sus aplicaciones exige un profundo estudio del comportamiento de los inóculos bajo condiciones controladas, debiendo demostrar su capacidad promotora de crecimiento en varias etapas, en primer lugar en condiciones axénicas, en el laboratorio, y después demostrarlo en experiencias de campo, en competencia con otros microorganismos. En esta última etapa, la fase más determinante es la colonización exitosa de la rizósfera, etapa en la que la planta seleccionará a aquellas cepas que le proporcionen más ventajas, como sintetizar factores de crecimiento, o movilizar nutrientes no disponibles para estas plantas (Jones et al., 1994) y además coexistirá con otras bacterias, con las que deberá establecer una relación. Es así, que existe una imperiosa necesidad de conocer el impacto del inóculo en las comunidades microbianas rizosféricas y en su estructura, tal como lo revelan Gilbert et al. (1993) y es también de importancia medir su posterior evolución en el tiempo con respecto a la comunidad original.

En tal sentido, la actividad microbiana en suelo rizosférico a nivel de campo, es considerado un parámetro clave en la determinación de la calidad biológica del suelo y para efectos del estudio realizado puede ser reflejo de condiciones físico-químicas óptimas para el desarrollo de los procesos metabólicos de microorganismos. Así, constituirá un buen indicador indirecto del desarrollo del cultivo de Phaseolus lunatus, elegido para el estudio. Además será índice directo del comportamiento microbiano luego de la aplicación de inoculantes naturales y químicos y de su efecto sobre la calidad y rendimiento del cultivo en términos de producción.
La actividad metabólica de los microorganismos está en función de los factores biofísicos, climáticos y del uso de la tierra y repercute en la tasa respiratoria, en tanto que la medida de la actividad deshidrogenasa, representa el metabolismo degradativo del suelo y es considerado un buen indicador por su sensibilidad y rápida respuesta a cambios en el manejo del suelo (Nannipieri, 1994).

Como precedente de este trabajo, Hernández \& Mayo (2002), evaluaron los parámetros de efectividad (peso seco de nódulos) e infectividad (número de nódulos) de algunas cepas de Rhizobium y Bradyrhizobium en dos variedades de pallar, Phaseolus lunatus var. criollo y var. sieva, a nivel de laboratorio y determinaron que las cepas de Bradyrhizobium PCYGIVN3 y PSNC4N2 eran efectivas, aunque esta última resultó menos infectiva, por presentar menor promedio de número de nódulos en las variedades de pallar evaluadas. El comportamiento de ambas cepas fue comparable al del control $\mathrm{N}+$, presentando diferencias altamente significativas respecto al control $\mathrm{N}$-, por lo que recomendaron su uso como inoculante en los cultivos mencionados, previa evaluación a nivel de campo. La conclusión anterior derivó en la evaluación de la actividad microbiana, del suelo de la rizósfera del cultivo de pallar tratado con los mismos inoculantes rizobianos, y se determinó su evaluación a través de la medida de la actividad microbiana por los métodos de actividad deshidrogenasa y producción de $\mathrm{CO}_{2}$, relacionándolo con la materia seca vegetal obtenida.

\section{Materiales y Métodos}

Diseño del campo experimental

El experimento se desarrolló en parcelas del campo El Olivar, en la localidad de Pachacamac, a 30 Km hacia el sur de la ciudad de Lima. Se utilizaron semillas del cultivo de Phaseolus lunatus var. sieva "pallar". Los tratamientos en el campo siguieron una distribución completa al azar, con tres repeticiones cada uno, un testigo sin inocular, uno con fertilización química nitrogenada $\mathrm{y}$ tres tratamientos con inoculantes rizobianos en soporte sólido: $\mathrm{T} 1$ con cepa de Bradyrhizobium PSNC4N2, T2 con Bradyrhizobium cepa PCYGIVN3, T3 con fertilización química nitrogenada N-P-K (60:60:60) y T4 con la interacción de dos cepas T1 y T2.

Muestreo y toma de muestra.

Se realizaron cinco muestreos, cada uno en diferente estado fenológico del cultivo, de acuerdo a la programación descrita en la Tabla 1. Para cada tratamiento evaluado se eligieron al azar tres plantas íntegras de tamaño promedio junto con la porción de suelo circundante a sus raíces. Las muestras colectadas fueron transportadas al laboratorio para su evaluación respectiva. 
Tratamiento de la muestra

Suelo.- El sistema radicular de cada planta fue separado de la masa de suelo circundante por agitación. Se procedió al retiro de todo material o residuo vegetal de la porción de suelo obtenida y luego de tamizar a un tamaño máximo de partícula de $500 \mu \mathrm{m}$, fue separado en frascos de vidrio con tapa y conservado a temperatura ambiente por $12 \mathrm{~h}$, hasta el análisis de producción de $\mathrm{CO}_{2}$ y de actividad de deshidrogenasa. Así también se determinaron el porcentaje de humedad y pH del suelo.

Tabla 1. Muestreo en diferentes estados fenológicos del cultivo de Phaseolus lunatus var. sieva.

\begin{tabular}{ccc}
\hline $\mathbf{N}^{\mathbf{o}}$ Muestreo & $\begin{array}{c}\mathbf{N}^{\mathbf{0}} \text { días después } \\
\text { de la siembra }\end{array}$ & Estado fenológico \\
\hline M - 1 & 39 & prefloración \\
M - 2 & 54 & floración \\
M - 3 & 71 & aparición de vainas \\
M - 4 & 96 & maduración \\
M - 5 & 131 & cosecha \\
\hline
\end{tabular}

Parte aérea.- Se determinó la materia seca vegetal y peso seco de vainas llevando la parte aérea de la planta a $60^{\circ} \mathrm{C}$ por 48 horas. Los resultados fueron expresados en gramos de peso seco / planta.

Determinación de la Actividad microbiana en la rizósfera

La producción de $\mathrm{CO}_{2}$ por los microorganismos del suelo se evaluó de acuerdo al método descrito por Anderson (1982) y por Ramos \& Zúñiga (2008). La actividad deshidrogenasa, fue determinada por reducción de sales tetrazolium, su extracción y cuantificación espectrofotométrica de acuerdo al método descrito por Casida (1977) y modificado por Roberge (1978) en la utilización de etanol como extrayente descrito por Ramos \& Zúñiga (2008). Los resultados se expresaron en $\mu \mathrm{g}$ de formazán·[g suelo seco $\cdot 24 \mathrm{~h}^{-1}$

Determinación de las poblaciones microbianas

Para la cuantificación de bacterias, mohos y levaduras, se realizaron diluciones sucesivas y se sembraron en medios de agar Plate Count para aerobios mesófilos viables y agar Czapeck para mohos y levaduras. Las bacterias aerobios mesófilos viables fueron incubadas a $30-35^{\circ} \mathrm{C}$ por $48 \mathrm{~h}$, mientras que, $\quad(\mathrm{P}=0.05)$.

para la enumeración de mohos y levaduras fue a 20$25^{\circ} \mathrm{C}$ por 5 días. Los resultados se expresaron en unidades formadoras de colonias por gramo de suelo seco $\left[\mathrm{UFC} \cdot \mathrm{g}^{-1}\right]$.

Análisis estadístico
Para cada ensayo, se realizó un análisis de variancia con un nivel de significación del 5\%. Diferencias significativas entre tratamientos fueron evaluadas mediante la prueba de diferencias de pares de medias de Tukey a un nivel de significación del 5\%. Alternativamente se utilizó la Prueba No Paramétrica de Kruskal Wallis con un nivel de confianza del $95 \%$, en caso de no cumplirse con los supuestos del ANOVA.

\section{Resultados y discusión}

La inoculación de semillas de pallar Phaseolus lunatus var. sieva con cepas efectivas de Bradyrhizobium, influye positivamente en la respiración y actividad deshidrogenasa de la rizósfera del cultivo, así como en el número total de bacterias. Etapa de Prefloración

Durante el estado fenológico de prefloración, no se registraron diferencias significativas en la producción de $\mathrm{CO}_{2}$ de los diferentes tratamientos, pero sí pudo apreciarse diferencias significativas $(\mathrm{p}<0.1)$ en la actividad deshidrogenasa (Tabla 2). Los tratamientos con inoculación presentaron mayor actividad microbiana que el control sin inocular y que el tratamiento con fertilización química nitrogenada. La interacción de cepas PSNC4N2 y PCYGIVN3 (T4) mostró la mayor actividad [101.21 $\mu \mathrm{g}$ formazán·g$\left.{ }^{1} \cdot(24 \mathrm{~h})^{-1}\right]$, seguido por el tratamiento inoculado con Bradyrhizobium cepa PSNC4N2 (T1) [100.02 $\mu \mathrm{g}$ formazán $\left.\cdot g^{-1} \cdot(24 \mathrm{~h})^{-1}\right]$, el inoculado con Bradyrhizobium cepa PCYGIVN3 (T2), [97.28 $\mu \mathrm{g}$ formazán $\cdot g^{-1} \cdot(24 h)^{-1}$ ], el Testigo (T0) [94.04 $\mu \mathrm{g}$

formazán $\left.\cdot g^{-1} \cdot(24 \mathrm{~h})^{-1}\right]$ frente el tratamiento que recibió fertilización química nitrogenada (T3) [con

Tabla 2. Producción de $\mathrm{CO}_{2}$, actividad deshidrogenasa (DHA) y población de aerobios mesófilos viables, mohos y levaduras en la rizósfera de pallar evaluada durante el estado fenológico de prefloración.

\begin{tabular}{|c|c|c|c|c|c|}
\hline \multirow{2}{*}{$\begin{array}{l}\text { Estado Fenológico } \\
\text { PREFLORACIÓN }\end{array}$} & \multicolumn{5}{|c|}{ Tratamientos } \\
\hline & T0 & T1 & T2 & T3 & T4 \\
\hline $\mathrm{CO}_{2} \quad\left[\mathrm{mg} \mathrm{CO}{ }_{2} \cdot \mathrm{g}-1 \cdot \mathrm{h}-1\right]$ & $0.082^{\mathrm{a}}$ & $0.086^{\mathrm{a}}$ & $0.081^{\mathrm{a}}$ & $0.080^{\mathrm{a}}$ & $0.074^{\mathrm{a}}$ \\
\hline $\begin{array}{l}\text { DHA } \\
\text { [ } \mu \mathrm{g} \text { formazán·g-1·(24h)-1] }\end{array}$ & $94.04^{\mathrm{b}}$ & $100.02^{\mathrm{b}}$ & $97.28^{b}$ & $67.06^{\mathrm{a}}$ & $101.21^{\mathrm{b}}$ \\
\hline $\begin{array}{l}\text { Aerobios mesófilos } \\
\text { [Ln(UFC:g-1)] }\end{array}$ & $19.25^{c}$ & $18.44^{\mathrm{bc}}$ & $17.32^{\mathrm{a}}$ & $17.43^{\mathrm{ab}}$ & $19.06^{\mathrm{bc}}$ \\
\hline $\begin{array}{l}\text { Mohos y levaduras } \\
\text { [Ln(UFC } \cdot g-1)]\end{array}$ & $10.28^{\mathrm{b}}$ & $10.60^{\mathrm{b}}$ & $10.83^{b}$ & $9.73^{\mathrm{a}}$ & $12.78^{\mathrm{c}}$ \\
\hline $\begin{array}{l}\text { Materia seca } \\
\text { [g/ planta] }\end{array}$ & $1.53^{\mathrm{a}}$ & $3.40^{\mathrm{a}}$ & $3.03^{\mathrm{a}}$ & $2.30^{\mathrm{a}}$ & $2.63^{\mathrm{a}}$ \\
\hline
\end{tabular}

a,b,c En cada fila, tratamientos seguidos por diferentes letras indican la existencia de diferencias significativas de acuerdo a la prueba de diferencias de pares de medias de Tukey $67.06 \mu$ g formazán $\left.\cdot g^{-1} \cdot(24 h)^{-1}\right]$.

Respecto a los recuentos de microorganismos, existen diferencias entre el tratamiento testigo (T0) y químico (T3), y el tratamiento de la interacción de cepas (T4) y el químico; en ambos casos, T3 mostró 
una disminución altamente significativa tanto para las poblaciones de aerobios mesófilos viables como para las mohos y levaduras.

En cuanto a materia seca de las plantas evaluadas (Tabla 2), se pudo observar que los tratamientos inoculados fueron mejores respecto al control sin inocular.

Etapa de Floración

Según Frioni (1999), la actividad microbiana rizosférica se ve incrementada desde la germinación de la semilla, dado el aporte de los microorganismos de la espermatósfera, los componentes de las envolturas del grano y por sus exudados; encontrando los máximos de estimulación durante las etapas de floración y/o fructificación, luego de lo cual se produce un descenso al iniciarse la senescencia de las raíces. En la Tabla 3, se muestran los valores de actividad microbiana y las poblaciones de aerobios mesófilos viables, mohos y levaduras encontradas durante este estado fenológico en la rizósfera del cultivo.

Tabla 3. Producción de $\mathrm{CO}_{2}$, actividad deshidrogenasa y población de aerobios mesófilos viables, mohos y levaduras en la rizósfera de pallar evaluada durante el estado fenológico de floración.

\begin{tabular}{|c|c|c|c|c|c|}
\hline \multirow{2}{*}{$\begin{array}{l}\text { Estado Fenológico } \\
\text { FLORACIÓN }\end{array}$} & \multicolumn{5}{|c|}{ Tratamientos } \\
\hline & T0 & T1 & $\mathbf{T 2}$ & T3 & T4 \\
\hline $\mathrm{CO}_{2}\left[\mathrm{mg} \mathrm{CO} \cdot \mathrm{CO}^{-1} \cdot \mathrm{h}^{-1}\right]$ & $0.049^{\mathrm{a}}$ & $0.088^{\mathrm{c}}$ & $0.058^{\mathrm{ab}}$ & $0.085^{\mathrm{bc}}$ & $0.121^{\mathrm{d}}$ \\
\hline $\begin{array}{c}\text { DHA } \\
{\left[\mu \mathrm{g} \text { formazán } \cdot \mathrm{g}^{-1} \cdot(24 \mathrm{~h})^{-1}\right]}\end{array}$ & $38.07^{\mathrm{a}}$ & $61.04^{\mathrm{b}}$ & $56.57^{\mathrm{b}}$ & $40.87^{\mathrm{a}}$ & $51.37^{\mathrm{ab}}$ \\
\hline $\begin{array}{l}\text { Aerobios mesófilos } \\
{\left[\mathrm{Ln}\left(\mathrm{UFC} \cdot \mathrm{g}^{-1}\right)\right]}\end{array}$ & $18.45^{\mathrm{ab}}$ & $18.84^{\mathrm{b}}$ & $18.19^{\mathrm{a}}$ & $18.68^{\mathrm{ab}}$ & $18.77^{\mathrm{b}}$ \\
\hline $\begin{array}{l}\text { Mohos y levaduras } \\
{\left[\operatorname{Ln}\left(\mathrm{UFC} \cdot \mathrm{g}^{-1}\right)\right]}\end{array}$ & $10.52^{\mathrm{a}}$ & $11.85^{\mathrm{ab}}$ & $11.94^{\mathrm{ab}}$ & $11.97^{\mathrm{ab}}$ & $12.28^{\mathrm{b}}$ \\
\hline Materia seca [g/ planta] & $6.60^{\mathrm{a}}$ & $8.27^{\mathrm{a}}$ & $7.07^{\mathrm{a}}$ & $7.20^{\mathrm{a}}$ & $10.43^{\mathrm{a}}$ \\
\hline
\end{tabular}

${ }^{a, b, c}$ En cada fila, tratamientos seguidos por diferentes letras indican la existencia de diferencias significativas de acuerdo a la prueba de diferencias de pares de medias de Tukey $(\mathrm{P}=0.05)$.

Se observó que el tratamiento de inoculación con la cepa Bradyrhizobium PSNC4N2 (T1) [0.088 mg $\mathrm{CO}_{2} \cdot \mathrm{g}^{-1} \cdot \mathrm{h}^{-1}$ y $61.04 \mu \mathrm{g}$ formazán $\left.\cdot \mathrm{g}^{-1} \cdot(24 \mathrm{~h})^{-1}\right]$ y el de interacción de cepas PSNC4N2 y PCYGIVN3 (T4) $\left[0.121 \mathrm{mg} \mathrm{CO}_{2} / \mathrm{g} * \mathrm{~h}\right.$ y 51.37 $\mu \mathrm{g}$ formazán $\left.\cdot g^{-1} \cdot(24 \mathrm{~h})^{-1}\right]$ fueron los que obtuvieron mayores incrementos de la actividad microbiana, resultando sus diferencias significativas respecto al testigo (T0) [0.049 mg $\mathrm{CO}_{2} \cdot \mathrm{g}^{-1} \cdot \mathrm{h}^{-1}$ y $38.07 \mu \mathrm{g}$ formazán $\left.\cdot \mathrm{g}^{-1} \cdot(24 \mathrm{~h})^{-1}\right]$. T4 además registró diferencias significativas con el tratamiento químico nitrogenado (T3) [0.085 mg CO $2 \cdot g^{-1} \cdot h^{-1}$ y $40.87 \mu$ g formazán $\left.\cdot g^{-1} \cdot(24 h)^{-1}\right]$. Así mismo, cabe señalar que el tratamiento de inoculación con la cepa Bradyrhizobium PCYGIVN3 (T2) también mostró una actividad deshidrogenasa importante $56.57 \quad \mu \mathrm{g}$ formazán $\cdot g^{-1} \cdot(24 h)^{-1}$, respecto a T3. De acuerdo al ensayo realizado, se obtuvo un incremento cercano al 35\% y superior al 100\% con la adición del inoculantes de Bradyrhizobium a la rizósfera del cultivo, respecto al tratamiento sin inocular, mostrando de manera clara su superioridad frente a la fertilización con compuestos comerciales nitrogenados, ya que con estos, la actividad microbiana aumentó en menos del 10\%. Esta misma tendencia puede evidenciarse en el rendimiento en materia seca. T4 presenta el mayor peso seco [10.43 g/ planta], seguido de T1 [8.27 g/ planta]; T3 presenta $[7.20 \mathrm{~g} /$ planta], mientras T0 presenta el menor valor [6.60 g/ planta].

Etapa de Aparición de Vainas

Para el estado fenológico de aparición de vainas, las diferencias entre tratamientos respecto a las variables evaluadas, no fueron significativas. Se puede resaltar que la actividad microbiana medida a través de la producción de $\mathrm{CO}_{2}$ fue más baja que en el muestreo anterior, encontrándose los mayores valores en el testigo y en el tratamiento de la interacción de cepas inoculadas. En cuanto a la actividad deshidrogenasa, fue relativamente constante en todos los tratamientos, ya que los valores promedio oscilaron entre 61.66 y $68.70 \mu \mathrm{g}$ formazán $\cdot \mathrm{g}^{-1} \cdot(24 \mathrm{~h})^{-1}$. Sin embargo, estos valores son superiores a los encontrados en la etapa de floración, lo que sugeriría la influencia de un factor externo, ya que las poblaciones de aerobios mesófilos viables, mohos y levaduras también bajaron en promedio una unidad logarítmica respecto al muestreo anterior (Tabla 4).

El tratamiento de la interacción de cepas inoculadas presentó el mayor contenido de materia seca, con $23.20 \mathrm{~g} /$ planta, T1 obtuvo también un alto contenido de materia seca [19.90 g/ planta], en tanto que T0 y T3 mostraron un mismo valor [16.15 g/ planta].

Tabla 4. Producción de $\mathrm{CO}_{2}$, actividad deshidrogenasa y población de aerobios mesófilos viables, mohos y levaduras en la rizósfera de pallar evaluada durante el estado fenológico de aparición de vainas.

\begin{tabular}{|c|c|c|c|c|c|}
\hline \multirow{2}{*}{$\begin{array}{c}\text { Estado Fenológico } \\
\text { APARICIÓN DE } \\
\text { VAINAS }\end{array}$} & \multicolumn{5}{|c|}{ Tratamientos } \\
\hline & T0 & T1 & $\mathbf{T} 2$ & T3 & T4 \\
\hline $\mathrm{CO}_{2}\left[\mathrm{mg} \mathrm{CO}_{2} \cdot \mathrm{g}^{-1} \cdot \mathrm{h}^{-1}\right]$ & 0.064 & 0.038 & 0.029 & 0.031 & 0.043 \\
\hline $\begin{array}{c}\text { DHA } \\
{\left[\mu \mathrm{g} \text { formazán } \cdot \mathrm{g}^{-1} \cdot(24 \mathrm{~h})^{-1}\right]}\end{array}$ & 68.70 & 61.66 & 64.61 & 64.97 & 63.34 \\
\hline $\begin{array}{c}\text { Aerobios mesófilos } \\
{\left[\mathrm{Ln}\left(\mathrm{UFC} \cdot \mathrm{g}^{-1}\right)\right]}\end{array}$ & 17.51 & 16.64 & 17.35 & 17.39 & 18.20 \\
\hline $\begin{array}{l}\text { Mohos y levaduras } \\
{\left[\operatorname{Ln}\left(\mathrm{UFC} \cdot \mathrm{g}^{-1}\right)\right]}\end{array}$ & 10.27 & 10.16 & 10.21 & 10.03 & 10.18 \\
\hline Materia seca [g/planta] & 16.15 & 19.90 & 11.25 & 16.15 & 23.20 \\
\hline
\end{tabular}


Etapa de Maduración de Vainas y Cosecha

Durante estas dos últimas etapas del cultivo de pallar, la tendencia de la actividad microbiana fue de incrementarse (Tablas 5 y 6), más aún antes de la cosecha, periodo en el cual la actividad deshidrogenasa aumentó alrededor del 300 y 400\% respecto a la etapa de floración para los tratamientos testigo (T0), fertilización química (T3) e interacción de cepas inoculadas (T4), en tanto que para los tratamientos inoculados T1 y T2, las actividades tuvieron un aumento cercano al 15\%. Esta diferencia coincidió con la presencia de fitonemátodes en la raíz, la cual fue evaluada cualitativamente. A nivel de laboratorio, se realizaron pruebas de actividad microbiana en la rizósfera de plantas con y sin fitonemátodes que fueron tratadas con diferentes inoculantes; luego del análisis estadístico, se demostró que la actividad en la rizósfera las plantas con fitonemátodes fue mayor que en las plantas que no los presentaban (datos no mostrados).

Tabla 5. Producción de $\mathrm{CO}_{2}$, actividad deshidrogenasa y población de aerobios mesófilos viables, mohos y levaduras en la rizósfera de pallar evaluada durante el estado fenológico de maduración de vainas

\begin{tabular}{|c|c|c|c|c|c|}
\hline \multirow{2}{*}{$\begin{array}{c}\text { Estado Fenológico } \\
\text { MADURACIÓN DE } \\
\text { VAINAS }\end{array}$} & \multicolumn{5}{|c|}{ Tratamientos } \\
\hline & T0 & T1 & $\mathbf{T} 2$ & T3 & T4 \\
\hline $\mathrm{CO}_{2}\left[\mathrm{mg} \mathrm{CO} \mathrm{CO}_{2} \cdot \mathrm{g}^{-1} \cdot \mathrm{h}^{-1}\right]$ & 0.074 & 0.067 & 0.066 & 0.076 & 0.077 \\
\hline $\begin{array}{c}\text { DHA } \\
{\left[\mu \mathrm{g} \text { formazán } \cdot \mathrm{g}^{-1} \cdot(24 \mathrm{~h})^{-1}\right]}\end{array}$ & 69.34 & 65.90 & 67.96 & 72.46 & 67.04 \\
\hline $\begin{array}{l}\text { Aerobios mesófilos } \\
\left.\left.\text { [Ln(UFC } \cdot \mathrm{g}^{-1}\right)\right]\end{array}$ & 18.21 & 16.72 & 18.14 & 18.82 & 17.17 \\
\hline $\begin{array}{l}\text { Mohos y levaduras } \\
{\left[\operatorname{Ln}\left(\mathrm{UFC} \cdot \mathrm{g}^{-1}\right)\right]}\end{array}$ & 10.24 & 11.18 & 11.98 & 9.99 & 10.15 \\
\hline Materia seca [g/ planta] & 13.15 & 20.60 & 18.90 & 18.00 & 22.80 \\
\hline
\end{tabular}

Tabla 6. Producción de $\mathrm{CO}_{2}$, actividad deshidrogenasa y población de aerobios mesófilos viables, mohos y levaduras en la rizósfera de pallar evaluada durante el estado fenológico antes de la cosecha

\begin{tabular}{|c|c|c|c|c|c|}
\hline \multirow{2}{*}{$\begin{array}{c}\text { Estado Fenológico } \\
\text { ANTES DE COSECHA }\end{array}$} & \multicolumn{5}{|c|}{ Tratamientos } \\
\hline & T0 & T1 & $\mathbf{T 2}$ & T3 & T4 \\
\hline $\mathrm{CO}_{2}\left[\mathrm{mg} \mathrm{CO} \cdot \mathrm{CO}^{-1} \cdot \mathrm{h}^{-1}\right]$ & 0.054 & 0.052 & 0.049 & 0.070 & 0.085 \\
\hline $\begin{array}{c}\text { DHA } \\
{\left[\mu \mathrm{g} \text { formazán } \cdot \mathrm{g}^{-1} \cdot(24 \mathrm{~h})^{-1}\right]}\end{array}$ & 143.45 & 70.09 & 71.03 & 180.30 & 172.78 \\
\hline $\begin{array}{l}\text { Aerobios mesófilos } \\
{\left[\operatorname{Ln}\left(\mathrm{UFC} \cdot \mathrm{g}^{-1}\right)\right]}\end{array}$ & 17.74 & 17.73 & 17.48 & 18.40 & 18.30 \\
\hline $\begin{array}{l}\text { Mohos y levaduras } \\
\quad\left[\operatorname{Ln}\left(\mathrm{UFC} \cdot \mathrm{g}^{-1}\right)\right]\end{array}$ & 11.31 & 12.17 & 11.67 & 11.23 & 13.13 \\
\hline Materia seca [g/ planta] & 9.50 & 21.15 & 18.45 & 14.90 & 11.90 \\
\hline
\end{tabular}

Las poblaciones de aerobios mesófilos viables, mohos y levaduras permanecieron relativamente constantes, por lo que no se registraron diferencias significativas. Respecto a la materia seca, durante el estado de maduración de vainas se observa aún la tendencia a favor de las plantas inoculadas con cepas de Bradyrhizobium, registrando valores de 18.9, 20.6 y $22.8 \mathrm{~g} /$ planta, correspondientes a los tratamientos T2, T1 y T4 respectivamente. Sin embargo esta tendencia se vio afectada durante el último muestreo; pues la presencia de fitonemátodes repercutió en la producción de la planta. Se observó que los valores de materia seca para el tratamiento de la interacción de cepas de Bradyrhizobium (T4) decrecieron, al igual que lo hicieron las plantas de los otros tratamientos afectados. Por otro lado, cabe resaltar que los tratamientos T1 y T2, que no se vieron afectados por nemátodos en las raíces, registraron los mayores promedios de materia seca [21.15 y 18.45 g/planta respectivamente].

Influencia de las condiciones ambientales en la actividad microbiana

La actividad deshidrogenasa incrementa de manera significativa como efecto de la interacción entre el contenido de agua y temperatura durante el periodo de preincubabción de la muestra (Brzezinska et al., 1998), por el contrario, Ross (1970) demostró que el secado del suelo al aire reducía la actividad deshidrogenasa a la mitad. Más aún, largos periodos de déficit de agua, afectan negativamente el proceso de fijación biológica de nitrógeno. Las sequías pueden inhibir la síntesis de proteínas de la nodulación, debido a la pérdida de leghemoglobina (Becana et al., 1986), disminuye la respiración de los nódulos (Aguirreolea \& Sánchez-Díaz, 1989), modifica el metabolismo del nódulo (Irigoyen et al., 1992) y/o afecta la actividad nitrogenasa (Schubert et al., 1995). Además, el stress hídrico puede acelerar la senescencia en los nódulos de las raíces como consecuencia de la reducción de su capacidad antioxidante y por el daño oxidativo de las biomoléculas resultantes (Gogorcena et al., 1995). A esto se suma la limitada tasa de difusión de nutrientes, de la matriz del suelo a la superficie absorbente, particularmente de fósforo, cuando la humedad del suelo declina (Pinkerton \& Simpson, 1986) afecta negativamente la nodulación y la fijación biológica de nitrógeno (O’Hara et al., 1988). Así mismo, Goicoechea et al. (2005) sugieren que la forma en que decrecen las actividades enzimáticas relacionadas al ciclo del ácido cítrico bajo condiciones de sequía, (como el caso de malato deshidrogenasa (MDH) e isocitrato deshidrogenasa (ICDH)), se debe a una reducción en el metabolismo aeróbico de los nódulos, 
Enero - Diciembre 2008

especialmente en aquellos de plantas no micorrízicas. El estrés hídrico puede reducir las tasas de respiración aeróbica (Aguirreolea \& Sánchez-Díaz, 1989) y estimula las rutas fermentativas (Irigoyen et al., 1992), además, disminuye la permeabilidad del oxígeno en la corteza del nódulo, restringiendo su acceso a los bacteroides (Witty et al., 1986).
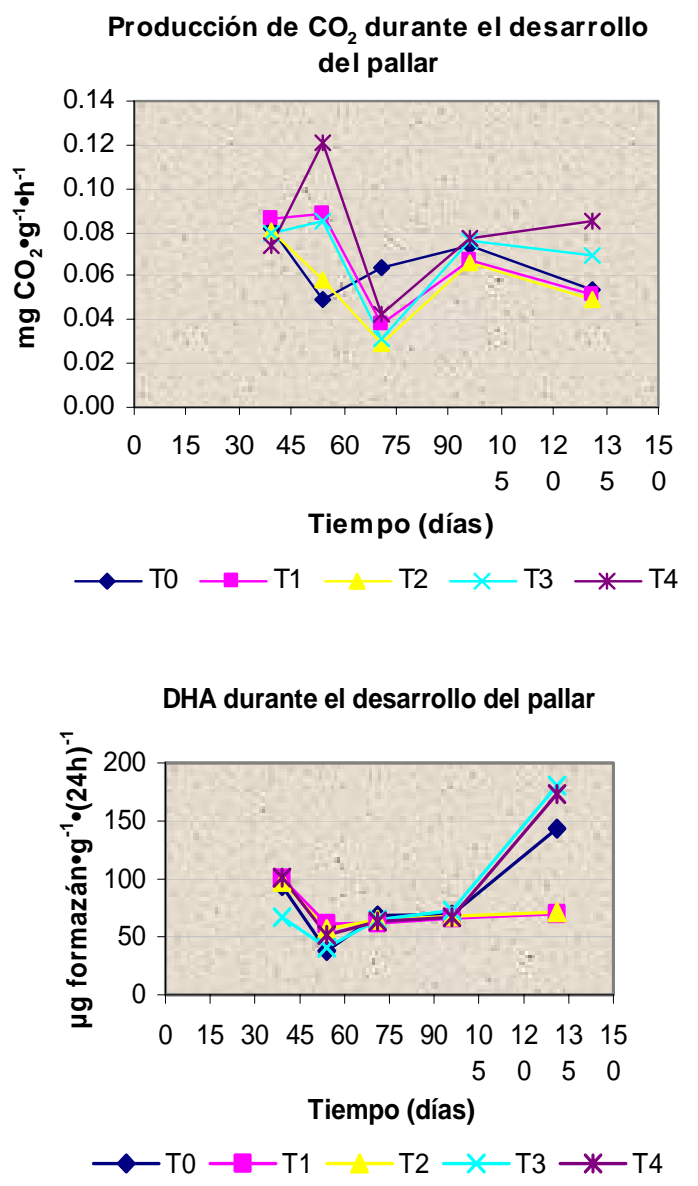

Figura 1. Producción de $\mathrm{CO}_{2}$ y actividad deshidrogenasa del suelo durante los diferentes estadíos del cultivo de pallar

Tomando la muestra directamente del campo, se tiene la ventaja de la frescura del material pero no provee ninguna clase de comparabilidad de condiciones, ni de resultados obtenidos por diferentes suelos. Esto podría explicar en parte la obtención de valores altos de DHA en la primera evaluación (Figura 1), etapa de prefloración, pues partimos de un porcentaje de humedad promedio del 15\% (Figura 2), además no debe ser despreciado el factor térmico, ya que el primer muestreo se dio a inicios de Marzo, tiempo en que la temperatura ambiental aún es cálida. Otros picos de actividad deshidrogenasa se produjeron durante las dos últimas evaluaciones (Figura 1), siendo más marcado su efecto en la quinta evaluación, si bien la humedad juega un rol importante en estos puntos de evaluación, debe recalcarse la presencia de fitonemátodes, que infectaron las raíces de muchas plantas hacia finales de su periodo vegetativo.

En general puede indicarse que los porcentajes de humedad del suelo al momento de realizar los ensayos de actividad microbiana a nivel de campo, estuvieron entre el 11\% y 18.07\% (Figura 2). Se registraron porcentajes de humedad entre 14.98 y $15 \%$ en el primer muestreo, entre 11.76 y $13.67 \%$ en el segundo muestreo, de 11.10 a $13.53 \%$ para el tercer muestreo, 15.53 a $18.07 \%$ para el cuarto muestreo y finalmente se obtuvieron valores de 12.70 a $14.70 \%$ para el último muestreo.

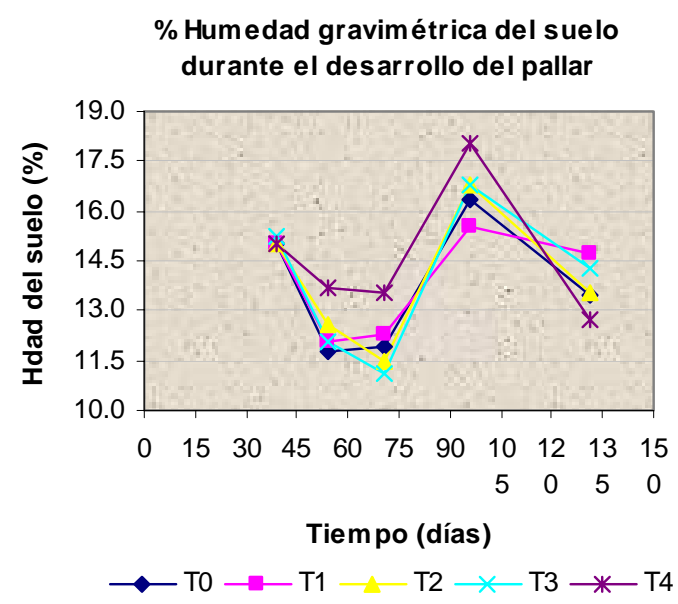

Figura 2. Porcentaje de humedad en la rizósfera de pallar vs. tiempo del cultivo.

En cuanto a los valores de $\mathrm{pH}$ de todas las muestras, estos se encontraron entre 7.1 y 7.8 , el valor más bajo corresponde a los tratamientos de plantas inoculadas con Bradyrhizobium cepa PSNC4N2 y Bradyrhizobium cepa PYG4N3, en tanto que el valor más alto fue registrado por el tratamiento Testigo (7.8), el tratamiento con fertilizante químico fue de 7.6 y el de la interacción con dos cepas fue de 7.4.

Respecto a la producción de $\mathrm{CO}_{2}$, pudo observarse que los picos de actividad se dieron hacia el periodo de floración para los tratamientos T2, T3 y T4 (Figura 1). Luego decae para la mayoría de los tratamientos aplicados, probablemente influenciado por el estado fenológico del cultivo y la humedad en suelo. Sin embargo, a diferencia de la medida de la actividad deshidrogenasa, no se vio alterada significativamente por la presencia de fitonemátodes en las raíces hacia finales del periodo vegetativo de la planta.

Cuantificación de microorganismos en la rizósfera de $\underline{P}$. lunatus var. sieva "pallar" bajo efecto de diferentes inoculantes

En la Figura 3. se muestra la dinámica de las poblaciones de bacterias aerobios mesófilos viables, y 
mohos y levaduras en la rizósfera del pallar bajo los diferentes tratamientos a través del tiempo.

Se observó que en general no se obtuvieron diferencias significativas, en el periodo de floración, solo se observaron diferencias entre los tratamientos T1 y T2 y entre T2 y T4 para el recuento de bacterias; mientras que para el recuento de mohos, se observan diferencias entre el tratamiento testigo y la interacción de cepas de Bradyrhizobium.

\section{Población de aerobios mesófilos viables} durante el desarrollo del pallar

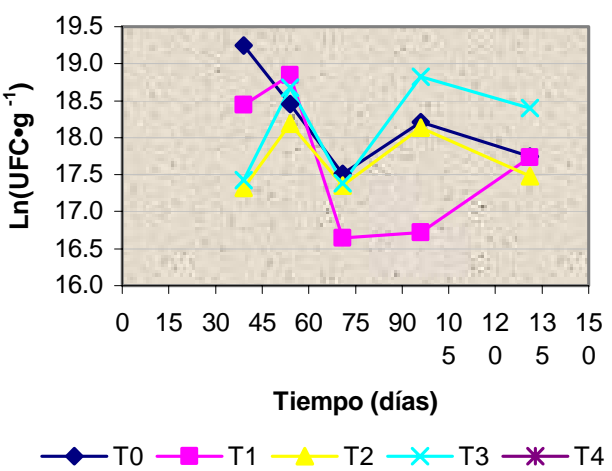

Población de mohos y levaduras durante el desarrollo del pallar

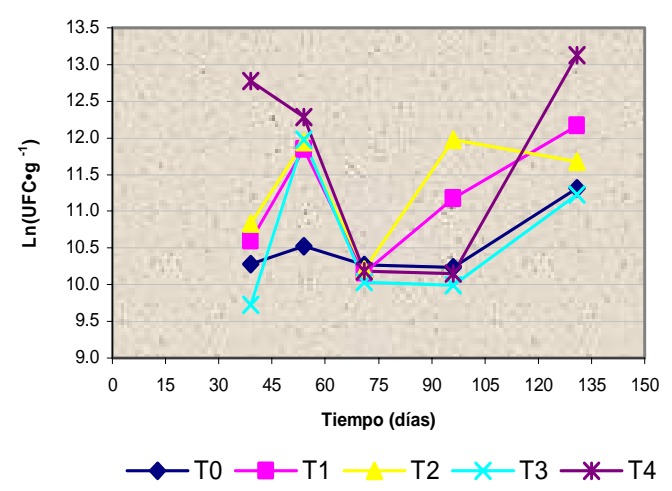

Figura 3. Cuantificación de la población aerobios mesófilos viables, mohos y levaduras durante el periodo de desarrollo del cultivo de pallar

Se observó que un cambio en la actividad microbiana no necesariamente reflejaba un cambio en el recuento realizado, lo cual podría indicar que la técnica de recuento en placa no resulta suficientemente sensible a los cambios producidos durante el desarrollo de un cultivo. La explicación puede deberse a que la determinación de células viables por conteo en placa generalmente produce valores demasiado bajos, pues su eficiencia relativa resulta en $0.1-5 \%$ de los microorganismos contados microscópicamente. Esto se debe a la selectividad del medio y a la incapacidad de diferentes células de formar colonias (Olsen \& Bakken, 1987; Mikell et al., 1996). Los microorganismos oligotróficos generalmente no crecen en medios con concentraciones de sustrato $>10 \mathrm{mg} \mathrm{L}^{-1}$ (Kuznetsov et al., 1979). Por otro lado, la adhesión a partículas de suelo causada por exopolisacáridos e incrustaciones en agregados, son la principal razón para la retención de microorganismos. Así, la liberación de microorganismos de ciertos tipos de suelo es muy dificultosa; además, las células liberadas tienden a rejuntarse con las partículas de suelo.

Dado este motivo, la utilización de diferentes propiedades bioquímicas para la caracterización de la actividad microbiana en la rizósfera de pallar resulta más ventajosa desde el punto de vista de comparación de tratamientos. La medición de dióxido de carbono es una estimación de la actividad y por lo tanto de la presencia microbiana; mientras que la actividad deshidrogenasa, dada su directa relación a los microorganismos viables totales, puede reflejar las bacterias, hongos y actinomicetos fisiológicamente activos (Thalman, 1968), pues se trata de una enzima intracelular que está asociada a células viables, además, su sensibilidad y rápida respuesta a cambios en el manejo del suelo resulta ventajosa.

\section{Conclusiones}

1. Los valores más altos de actividad microbiana medida por la producción de $\mathrm{CO} 2$ fueron obtenidos durante el estado de floración de $P$. lunatus var. sieva. (54 días de la siembra). La actividad deshidrogenasa fue superior en la última evaluación realizada (131 días de la siembra).

2. Los valores más altos de actividad microbiana medida por producción de CO2 fueron obtenidos durante el estado de floración de $P$. lunatus var. sieva. En tanto que la actividad deshidrogenasa fue superior en la última evaluación realizada (131 días de la siembra). Durante la floración, se observaron los mayores valores de actividad microbiana en la rizósfera de pallar inoculado con Bradyrhizobium cepa PSNC4N2 (T1) [0.088 mg CO2·g-1·h-1 y $61.04 \mu \mathrm{g}$ formazán·g-1·(24h)-1] y con el tratamiento de interacción de cepas de Bradyrhizobium PSNC4N2 y PCYGIVN3 (T4) [0.121 mg CO2·g-1·h-1 y 51.37 g formazán·g1.(24h)-1]. Dichos tratamientos obtuvieron diferencias significativas respecto al testigo (T0) [0.049 mg CO2·g-1·h-1 y 38.07 ug formazán·g$1 \cdot(24 \mathrm{~h})-1]$, en tanto que el tratamiento de la interacción de cepas inoculadas (T4) registró además diferencias significativas con el tratamiento químico nitrogenado (T3) [0.085 mg CO2·g-1·h-1 y 40.87 ug formazán·g-1·(24h)-1].

3. Respecto a los recuentos de las poblaciones de aerobios mesófilos viables, mohos y levaduras, se 
encontraron algunas diferencias significativas entre tratamientos durante las etapas de prefloración y floración del cultivo de pallar.

4. En materia seca vegetal, el tratamiento de la interacción de cepas de Bradyrhizobium (T4) y el inoculado solo con Bradyrhizobium cepa PSNC4N2 (T1) presentaron los valores más altos durante los 54 y 96 días después de la siembra (periodo comprendido entre los estados de floración y madurez de vainas), mientras que los tratamientos testigo (T0) y de fertilización nitrogenada (T3) presentaron valores inferiores.

5. La interacción de cepas de Bradyrhizobium PSNC4N2 y PCYGIVN3 (T4) presenta un impacto positivo en la actividad microbiana de las comunidades rizosféricas del cultivo de Phaseolus lunatus var. sieva y en el incremento de materia seca del mismo, por lo que se sugiere su uso como biofertilizante.

\section{Agradecimientos}

CONCYTEC - PROCYT No 207 - 2005. Al Laboratorio de Ecología Microbiana y Biotecnología "Marino Tabusso"- UNALM (Subcuenta Biología 111 de la Fundación para el Desarrollo Agrario). Al Programa de Leguminosas de la Facultad de Agronomía - UNALM. Al profesor Mg. Sc. Abelardo Calderón del Laboratorio de Fisiología Vegetal, al Blgo. Juan Mayo y a Carlos García por las facilidades prestadas.

\section{Literatura citada.}

Aguirreolea J. \& Sánchez-Díaz M. 1989. CO2 evolution by nodulated roots in Medicago sativa $\mathrm{L}$. under water stress. J Plant Physiol. 134: 598-602.

Anderson J. 1982. Soil Respiration. En: Page A., Miller R. y Kenney D. (Eds). Methods of soil analysis. Part II. Chemical and microbiological properties. 2nd ed. American Society of Agronomy. Number 9. Madison, Wisconsin, USA. 831-871.

Andreeva I., Redkina T. \& Izmaliov S. 1993. The involvement of indoleacetic acid in the estimulation of Rhizobium-legume symbiosis by Azospirillum brasilensis. Russian J.Pl Phys. 40: 901-906.

Becana M., Aparicio-Tejo P., Peña J., Aguirreolea J. \& Sánchez-Díaz M. 1986. N2 fixation (C2H2-reducing activity) and leghaemoglobin content during nitrate and water stress-induced senescence of Medicago sativa root nodules. J Exp Bot. 37:597-605.

Brzezinska M., Stepniewska Z. \& Stepniewski W. 1998. Soil oxygen status and dehydrogenase activity. Soil Biol. Biochem. 30:1783-1790.

Casida L. 1977. Microbial metabolic activity in soil as measured by dehydrogenase determinations. Applied and Environmental Microbiology. 34: 630-636. 17.

Defreitas J., Gupta V. \& Germida J. 1993. Influence of Pseudomonas syringae R25 and P. putida R105 on the growth and N2 fixation (acetylene reduction activity) of pea (Pisum sativum L.) and field bean (Phaseolus vulgaris L.). Biol. Fertil. Soils. 16: 215-220.
Frioni L. 1999. Procesos Microbianos. Editorial de la Fundación Universidad Nacional de Río Cuarto. Argentina.

Gilbert G., Parke J., Clayton M. \& Handelsman J. 1993. Effects of an introduced bacterium on bacterial communities on roots. Ecology. 74: 840-854.

Gogorcena Y., Iturbe-Ormaetxe I., Escuredo P. \& Becana M. 1995. Antioxidant defenses against activated oxygen in pea nodules subjected to water stress. Plant Physiol. 108: 753-759.

Goicoechea M., Lacombe E., Legay S., Mihaljevic S., Rech P., Jauneau A., Lapierre C., Pollet B., Verhaegen D., Chaubet-Gigot N. \& Grima-Pettenati J. 2005. EgMYB2, a new transcriptional activator from Eucalyptus xylem, regulates secondary cell wall formation and lignin biosynthesis. Plant J. 43: 553-567.

Hernández J. \& Mayo J. 2002. Infectividad y efectividad de cepas nativas de rizobios aislados de la provincia de Ica en Phaseolus lunatus (pallar) var. criollo iqueño y var. sieva. Universidad Nacional San Luis Gonzaga de Ica. Tesis para optar el título de Biólogo.

Irigoyen J., Emerich D. \& Sánchez-Díaz M. 1992. Phosphoenolpyruvate carboxylase, malate and alcohol dehydrogenase activities in alfalfa (Medicago sativa) nodules under water stress. Physiol Plant. 84: 61-6.

Jones D., Edwards A., Donachie K. \& Darrah P. 1994. Role of proteinaceous aminoacids realised inroot exudates in nutrient acquisition from the rhizosphere. Plant and Soil. 158: 183-192.

Kuznetsov S., Dibinina G. \& Lapteva N. 1979. Biology of oligotrophic bacteria. Annual Reviews of Microbiology. 33: 377-387.

Mikell A., Smith C. \& Richardson J. 1996. Evaluation of media and techniques to enumerate heterotrophic microbes from karst and sand aquifer springs. Microbial Ecology. 31: 115-124.

Nannipieri P. 1994. The potential use of soil enzymes as indicators of productivity, sustainability and pollution. En: Pankhurst C., Double B., Gupta V., Grace P. (Eds.). Soil Biota: Managment in Sustainable Farming Systems. CSIRO, Melbourne. : 238-244.

O’Hara G., Dilworth M., Bookerd N. \& Parkpian P. 1988. Iron deficiency specifically limits nodule development in peanut inoculated with Bradyrhizobium spp. New Phytologist. 108: 51-57.

Olsen R. \& Bakken L. 1987. Viability of soil bacteria: optimization of plate-counting technique and comparison between total counts within different size groups. Microbial Ecology. 13: 59-74.

Pinkerton A. \& Simpson J. 1986. Interactions of surface drying subsurface nutrients affecting plant growth on acidic soil profile from an old pasture. Aust J Exp Agric. 26: 681-686.

Probanza A., Acero N., Ramos B. \& Gutierrez Mañero F. 1997. Effects of european alder (Alnus glutinosa L. Gaertn) rhizobacteria on nodular metabolism and root development. Pl Growth Reg. 22: 145-149.

Ramos E. \& Zúñiga D. 2008. Efecto de la humedad, temperatura y $\mathrm{pH}$ del suelo en la actividad microbiana a nivel de laboratorio. Ecología Aplicada. Vol.7 (En prensa).

Roberge M. 1978. Methodology of soil enzyme measurement and extraction: catalase En: Soil Enzymes, ed. Burns R., p. 344. Academic Press, London. 
Ross D. 1970. Effects of storage on dehydrogenase activities of soils. Soil Biology \& Biochemistry. 2: 5561.

Schubert S., Serraj R., Plier-Balzer E. \& Mengel K. 1995. Effect of drought stress on growth, sugar concentrations and amino acid accumulation in N2-fixing alfalfa (Medicago sativa). J Plant Physiol. 145: 541-546.
Thalman A. 1968. Zur Methodik de Bestimmung der Dehydrogenase Aktivität im Boden Mittels Triphenyltetrazoliumchlorid (TTC). Landwirsch. Forsch. 21: 249-257.

Witty J., Minchin F., Skøt L. \& Sheehy J. 1986. Nitrogen fixation and oxygen in legume root nodules. Oxf Surv Plant Mol Cell Biol. 3: 275-314.

${ }^{1}$ Laboratorio de Ecología Microbiana y Biotecnología Marino Tabusso. Dpto. de Biología, Universidad Nacional Agraria La Molina. 2005. Av La Molina s/n. Lima 12 - Perú. dzuniga@lamolina.edu.pe, web: www.lamolina.edu.pe/lmt 